Paper submitted in:

EIWAC. The Enri International Workshop on ATM/CNS. 14th -16th

November, 2017, Tokyo, Japan

Available in: https://www.enri.go.jp/eiwac/eiwac_2017/special-site_eiwac2017/

program.html

(C) The Author(s)

Funding: Partially supported by project TBO-MET project (https://tbomet-h2020.com/), which has received funding from the SESAR JU under grant agreement No 699294 under European Union's Horizon 2020 research and innovation programme.

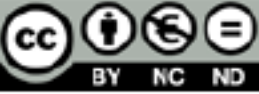

This work is licensed under a Creative Commons Attribution-NonCommercial-

NoDerivatives 4.0 International License. 


\title{
[EN-A-073] Robust Optimal Trajectory Planning under Uncertain Winds and Convective Risk
}

\author{
${ }^{+}$Daniel González-Arribas*, Manuel Soler*, Manuel Sanjurjo-Rivo*, Javier García-Heras*, Daniel Sacher ${ }^{\mathbb{T}}$, \\ Ulrike Gelhardt", Juergen Lang ${ }^{\Uparrow}$, Thomas Hauf $f^{\ddagger}$, and Juan Simarro ${ }^{\S}$ \\ *Escuela Politécnica, Area of Aerospace Engineering, Universidad Carlos III de Madrid, Leganés, Madrid, 28911 \\ Email: dangonza, masolera, msanjurj@ing.uc3m.es \\ ๑MeteoSolutions GmbH. Sturzstraße 45, D-64285 Darmstadt (Germany) \\ Email: ulrike.gelhardt, daniel.sacher, juergen.lang@meteosolutions.de \\ $\ddagger$ Retired Prof. for meteorology at the Leibniz University Hannover. Scientific Advisor of TBO-Met Project. \\ Email: hauf@muk.uni-hannover.de \\ $\S$ Agencia Estatal de Meteorología (AEMET) \\ Email: jsimarrog@aemet.es
}

\begin{abstract}
The existence of significant uncertainties in the models and systems required for trajectory prediction represent a major challenge for Trajectory-Based Operations concept. Weather can be considered as one of the most relevant sources of uncertainty. Understanding and managing the impact of these uncertainties is necessary in order to increase the predictability of the ATM system. We present preliminary results on robust trajectory planning in which weather is assumed to be the unique source of uncertainty. State-of-the-art forecasts from Ensemble Prediction Systems are used as input data for the wind field and to calculate convective risk. The term convective area is defined here as an area within which individual convective storms may develop, i.e., a necessary (though not sufficient) condition. An ad-hoc robust optimal control methodology is presented. A set of Pareto-optimal trajectories is obtained for different preferences between predictability, convective risk and average efficiency.
\end{abstract}

\section{INTRODUCTION}

A major challenge for Trajectory-Based Operations (TBO) is the existence of significant uncertainties in the models and systems required for trajectory prediction. Understanding and managing the impact of these uncertainties is necessary in order to increase the predictability of the ATM system. In turn, predictability and robustness improvements in trajectories will produce gains in the high level goals (capacity, efficiency, safety, and environmental impact) pursued within a modernized ATM system. Some examples of relevant uncertainty sources are: 1) meteorological uncertainty; 2) uncertainty in the aircraft performance model [1];3) uncertainty in initial mass[2] and other parameters and 4) uncertainty in the aircraft intent [3]. In this paper, the focus is on the former, i.e., meteorological uncertainty, one of the most important sources of uncertainty that affect the ATM system. Indeed, the recently granted SESAR ER TBO-Met Project ${ }^{1}$ focuses on the analysis of meteorological uncertainty coming from the following two sources: 1) wind, and 2) convective regions. While we won't consider these additional uncertainty sources, we will note that out methodology could be extended to include them.

The main contribution of this paper is to extend the methodology for robust route optimization in [4], [5] to the considera-

${ }^{1}$ TBO-MET project (https://tbomet-h2020.com/) has received funding from the SESAR JU under grant agreement No 699294 under EU's Horizon 2020 research and innovation programme. Consortium members are UNIVERSITY OF SEVILLE (Coordinator), AEMET (Agencia Española de Meteorología), METEOSOLUTIONS GmbH, PARIS-LODRON-UNIVERSITAT SALZBURG, and UNIVERSIDAD CARLOS III DE MADRID. tion of convection risk. The focus is on the pre-tactical level (in this context, around 3 hours before departure). We make use of Ensemble Prediction Systems and optimal control techniques. Figure I sketches the intended methodology for the Trajectory planning problem in TBO-Met Project. The pre-tactical level is represented in the left hand side of the figure, considering both wind uncertainty and convective phenomena.

Convective regions are defined as areas within which individual convective storms may develop. The latter comprise individual storm cells, multi-cells, mesoscale convective complexes and squall lines. Convective storms need a trigger mechanism and the onset and the location of those individual storms is impossible to forecast at the flight planning level. Nevertheless, one can obtain forecasts with some characteristics that act as necessary conditions (however not sufficient) for the formation of storms, and that combined can provide a probability of convection, i.e., an indicator of convection risk than can be used for trajectory planning.

Numerical Weather Prediction (NWP) centers developed Ensemble Prediction Systems (EPS) in order to provide probabilistic meteorological forecasts in addition to deterministic predictions. They seek to provide an estimation of the uncertainty that is inherent to the NWP process [6], a task that cannot be achieved with deterministic forecasting. In an EPS, several runs of the NWP model are launched with different characteristics in order to produce a set of (typically) 10 to 50 different forecasts or "members" of the ensemble. We refer to [7] for a review of the status of NWP as well as the relevance of EPS in a wider meteorological context. 


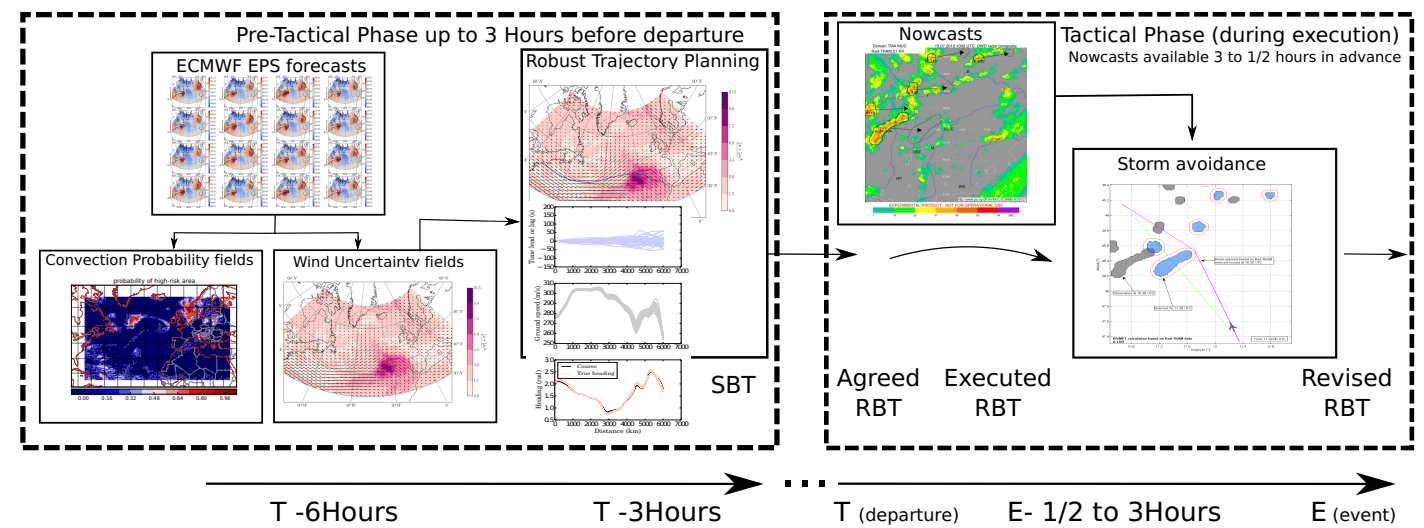

Figure 1. TBO-Met Trajectory Planning Methodology for both pre-tactical and tactical levels. Recall that the present paper focuses on the pre-tactical level.

The ATM research community has recently started to use EPS in order to study the predictability of flight plans and the sensitivity to weather prediction uncertainty. The main research effort in this direction has been undertaken within IMET, a SESAR WP-E project. It sought to develop a "probabilistic trajectory prediction" (PTP) system, where a deterministic TP is run once for each member in order to produce a trajectory ensemble. Preliminary results of this project were presented in [8] and a follow-up publication [9] showed how the information obtained with this approach could be used to improve decision-making at the pre-tactical level. Outside IMET, e.g., in [10] the authors present an analysis of the impact of uncertainty in average wind on final fuel consumption. In [4], [5] we developed (within the framework of SESAR's TBO-Met project) a robust approach to aircraft trajectory planning under wind uncertainty using EPS. In parallel to the latter, an approach based on Dynamic Programming for aircraft trajectory planning under wind uncertainties (and also using EPS) has been recently published in [11]. All in all, the focus of all these works is on wind uncertainty. To the best of authors' understanding, the inclusion of convective indicators in robust flight planning is an unexplored field. Thus, we go beyond the state of the art by extending the approach in [4], [5] to the consideration of convection.

The paper is structured as follows: we introduce convection and its associated indicators in Section II. The robust optimal control methodology is presented in Section III. In section IV we present a case study, including the simulation results and a discussion. Finally, some conclusions are drawn in Section V.

\section{Convection}

\section{A. Convection Indicators}

Within this paper it is attempted to delimit high-risk areas due to deep convection and their respective uncertainty. The term convective area is defined here as an area of potentially developing storms. The latter comprise individual storm cells, multi-cells, mesoscale convective complexes and squall lines. The onset and the location of those individual storms are difficult to evaluate for the time being and impossible to determine in many cases. Favourable environmental characteristics and conditions for certain types, however, are known, e.g.:

- A squall line (at least in Central Europe) very often develops several hundred kilometres ahead of and parallel to an approaching cold front. It is initiated and recognized by a boundary convergence line. Many such lines often occur approx.10m km apart, but not all of them necessarily develop into a squall line, though some of them do.

- Air mass storms preferably develop in the afternoon. The onset time of first shallow clouds and the development of deep convective clouds can be forecasted by standard meteorological procedures.

- Moderate mid-level shear enhances the storm strength, while too strong shear and no-shear environments are more likely related to weak storms

- Long-lived storms are linked to the renewal and generation of new cells immediately ahead of a mature cell.

- Storms embedded in a cold front are out of scope of this study as they can be forecasted very well by synoptic forecasts of low pressure systems.

- The structure of the environmental temperature profile (temp) allows deriving certain features of the storm. Maritime dominated storms reveal a temp close to the moist-adiabatic implying weak updrafts, while continental storms exhibit more potential energy to be released. The latter is defined by the area between moist adiabatic and the temperature profile.

- Environmental characteristics are used to derive empirically a range of convective indices.

Important to note is that the above characteristics are necessary conditions, but they do not allow the forecast of the precise location and onset. Convective storms need a trigger mechanism. In order to precisely forecast a storm, we therefore need to forecast the trigger mechanisms like e.g. boundary convergence lines, tropospheric gravity waves, mountains or surface temperature inhomogeneity.

From the above we conclude that we need an indicator to describe the necessary precondition for the potential development of convection and an indicator which comprises the 
essential activator in order to develop a storm which has to be avoided by aircraft. As described below this will be done by using a combination of two convection indicators Total Totals Index and Convective Precipitation, which are available by the EPSs. When both indicators exceed certain thresholds for a high number of EPS members, the grid point is assumed to lie within the zone of high probability (low uncertainty) of convection which can be interpreted as a no-fly-zone. If only one criterion is fulfilled for a high number of EPS members the grid point is located in a region of convective uncertainty. The boundary of uncertainty areas will delimit convective regions.

Those convective areas may have a persistence or life time of up to 60 hours. Carbone et al. [12] and previous studies investigated precipitation episodes and found much longer life times of those episodes, respectively travelling convective regions, than those of the individual storms de-veloping within. Here we pursue similar thoughts. Convective regions are perceived as areas with a high weather risk, the latter given by always occurring and unpredictable individual storms. Convective regions, therefore, must not necessarily be avoided but require a higher weather situation awareness by pilots and controllers. Also, trajectories passing through a convective area are subject to diversions resulting in increased flight duration and delays. Thus the intersection of a trajectory with a convective region does not imply, as already said above, that the whole area has to be circumnavigated, but rather that delays have to be expected. The dimension of the latter depends, among other factors, on the type of storms embedded in the convective area, density of cells, their orientation, the size of gaps separating the storms and the time of onset.

We decided to combine two indicators for convection:

1) Total Totals Index (TT): ${ }^{2}$ The sum of the vertical totals (VT) $V T=T_{850}-T_{500}$ (temperature gradient between 850 $\mathrm{hPa}$ and $500 \mathrm{hPa}$ ) and the cross totals $(C T) C T=T_{d_{850}}-T_{500}$ (moisture content between $850 \mathrm{hPa}$ and $500 \mathrm{hPa}$ by subtracting the temperature in $500 \mathrm{hPa}$ from dew point temperature in 850 $\mathrm{hPa})$. As a result, $T T$ accounts for both static stability and $850 \mathrm{hPa}$ moisture, but would be unrepresentative in situations where the low-level moisture resides below the $850 \mathrm{hPA}$ level. In addition, convection may be inhibited despite a high $T T$ value if a significant capping inversion is present. $V T=40$ is close to dry adiabatic for the $850-500 \mathrm{hPa}$ layer. However, $V T$ generally will be much less, with values around 26 or more, representing sufficient static instability (without regard to moisture) for thunderstorm occurrence. $C T>18$ often is necessary for convection, but it is the combined Total Totals Index that is most important. The risk of severe weather activity is operationally defined as follows (see also [13]):

- 44-45: isolated moderate thunderstorms

- 46-47: scattered moderate / few heavy thunderstorms

- 48-49: scattered moderate / few heavy / isolated severe thunderstorms

- 50-51: scattered heavy / few severe thunderstorms and

${ }^{2}$ attributable to National Weather Service Louisville, KY: http://www.weather.gov/lmk/indices, accessed July 25, 2016. isolated tornadoes

- 52-55: scattered to numerous heavy / few to scattered severe thunderstorm / few tornadoes

- >55: numerous heavy / scattered severe thunderstorms and scattered tornadoes

2) Convective Precipitation $(C P):{ }^{3}$ an estimation of the precipitation coming from convective clouds. The total precipitation is the sum of the so-called large-scale precipitation and the convective precipitation.

The moist convection scheme is based on the mass-flux approach and represents deep (including cumulus congestus), shallow and mid-level (elevated moist layers) convection. The distinction between deep and shallow convection is made on the basis of the cloud depth (i $200 \mathrm{hPa}$ for shallow). For deep convection the mass-flux is determined by assuming that convection removes Convective Available Potential Energy (CAPE) over a given time scale. The intensity of shallow convection is based on the budget of the moist static energy, i.e. the convective flux at cloud base equals the contribution of all other physical processes when integrated over the sub-cloud layer. Finally, mid-level convection can occur for elevated moist layers, and its mass flux is set according to the large-scale vertical velocity. The scheme, originally described in Tiedtke [14], has evolved over time and amongst many changes includes a modified entrainment formulation leading to an improved representation of tropical variability of convection [15], and a modified CAPE closure leading to a significantly improved diurnal cycle of convection [16].

\section{B. Calculation of probability of convention/clear air}

In order to fulfil the desired requirements, the following data processing for convection will be provided which can be applied individually or in a processing chain:

- Grid-based output of the Total Totals Index and the Convective Precipitation: Using the ECMWF-ENS data, both convective indicators, TT and $\mathrm{CP}$ are given. The results of this workflow are the TT and $\mathrm{CP}$ for each member at the horizontal nodes of the desired sub-grid.

- Ensemble-based probability of convection / clear air for each grid point: With regard to flight trajectories it is important to delimit regions of uncertain weather conditions from regions where the forecast is more reliable. Convective regions of high uncertainty can then be defined as those areas where neither convection nor clear air can be safely predicted. So, the calculation of two quantities is suggested:

- Probability of convection

- Probability of clear air

Probability of convection: The ensemble-based probability of convection is the fraction of ensemble members with values above the given thresholds $T T_{H}$ and $c p_{H}$ for all $T T$ and $c p$ of the ensemble members. For $T T_{H}$ we suggest one of the threshold values given in the list above. For $c p_{H}$ we

${ }^{3}$ ECMWF, Reading, UK, accessed July 25, 2016: http://www.ecmwf.int/en/research/modelling-and-prediction/atmosphericphysics. 


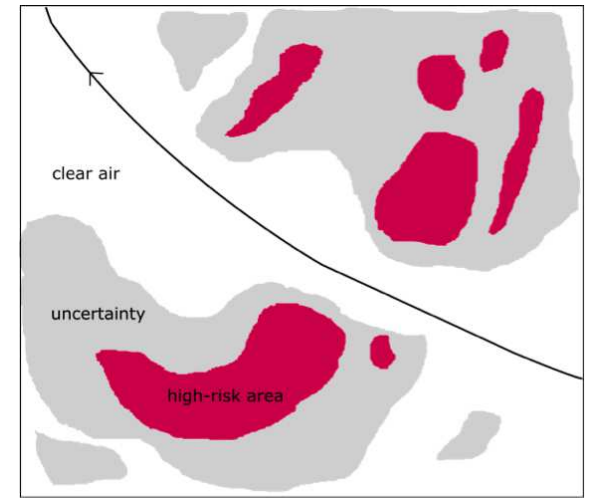

Figure 2. Schematic illustration of the suggested classification of the focused area into 3 different zones: clear air (white), high-risk areas (pink) and uncertainty (grey).

suggest 0 ; which means that any given amount of convective precipitation originates from convective events:

$$
p_{c}=\frac{N_{c}}{N},
$$

where $\mathrm{N}$ is the numbers of ensemble members, $N_{c}=\sum_{i=1}^{N} i$, and so that $T T_{i}>T T_{H} \wedge c p_{i}>c p_{H}$.

Probability of clear air: Value that can show regions of clear air with low uncertainty:

$$
p_{n c}=\frac{N_{n c}}{N},
$$

where $\mathrm{N}$ is the numbers of ensemble members, $N_{n c}=\sum_{i=1}^{N} i$, and so that $T T_{i} \leq T T_{H} \wedge c p_{i} \leq c p_{H}$.

Considering both values $p_{c}$ and $p_{n c}$ at each grid node we are able to divide the focused area into 3 zones (see Figure 2 for an schematic):

1) Convective zones i.e. high-risk areas with low uncertainty,

2) Clear air zones with low uncertainty,

3) Zones with high uncertainty.

With these two parameters $\left(p_{c} \& p_{n c}\right)$ further postprocessing (e.g., classifications as described above) can be done.

High-risk areas for each ensemble member: : In order to get high-risk areas where each zone is based on the individual prediction of a single ensemble member, we look at the forecasted values of $T T_{i}$ and $c p_{i}$ at each horizontal grid node. In analogy to the ensemble-base probability of convection, we define a high-risk area for an ensemble member as an area where the following condition is fulfilled at each grid point: $T T_{i}>T T_{H} \vee c p_{i}>c p_{H}$.

That means that a high-risk area is delimited by the regions of low uncertainty which include the regions of high probability of convection. As the Total Totals Index is a smooth field, we suppose that we get clear structures of convective zones as well. Otherwise morphological operations can be applied to the generated field in order to eliminate unreliable singularities in the convective zones.

\section{Robust Optimal Control}

The class of dynamical systems that we will consider what [17] calls a tychastic dynamical system. We denote the state vector by $\mathbf{x} \in \mathbb{R}^{n}$, the control vector by $\mathbf{u} \in \mathbb{R}^{m}, t \in \mathbb{R}$ is the independent variable (usually time) and the uncertain parameters are a continuous constant random variable $\xi: \Omega \rightarrow$ $\mathbb{R}^{q}$. The dynamics of the system are given by the function $f: \mathbb{R}^{n} \times \mathbb{R}^{m} \times \mathbb{R}^{q} \times \mathbb{R} \rightarrow \mathbb{R}^{m}$, such that:

$$
\frac{d}{d t} \mathbf{x}(\omega, t)=f(\mathbf{x}(\omega, t), \mathbf{u}(\omega, t), \xi(\omega), t)
$$

where $\omega \in \Omega$ is the sample point on the underlying abstract probability space. Thus, for each possible realization of the random variable $\xi(\omega)$, the trajectory will follow the deterministic differential equation (III-B) ${ }^{4}$. To emphasize the dependence of the trajectories on the random variables, we will use the notation $\mathbf{x}(\omega, t)$ and $\mathbf{u}(\omega, t)$

In order to fully determine the trajectory, we will need a control or guidance law in addition to the realization of the uncertain parameters $\xi$. We will discuss this topic in Section III-C; consider, meanwhile, a general control law

$\mathbf{u}(\omega, t)=\mathbf{u}_{L}(t, \mathbf{x}(\omega, t))$

\section{A. Stochastic quadrature rules}

The first component of this methodology is a stochastic quadrature rule: a finite set of quadrature points $\left\{\xi_{k}\right\}$, $k \in\{1, \ldots, N\}$ and weights $\left\{w_{k}\right\}, k \in\{1, \ldots, N\}$, such that we can build an approximation to the stochastic integral $I=\int_{\Omega} g(\xi(\omega)) d \omega$ with the sum:

$$
Q g=\sum_{k=1}^{N} w_{k} g\left(\xi_{k}\right)
$$

where $g(\xi)$ is an arbitrary function. Basic statistical quantities, such as averages and variances, can be obtained with this integral by the corresponding function choices. There are a number of approaches with different approximation techniques that can provide a stochastic quadrature rule: Monte Carlo methods, Quasi-Monte Carlo methods (see e.g. [18], [19]), Cubature techniques (see e.g.,[20] and [21]. In [17]), Stochastic Collocation of Generalized Polynomial Chaos $(g P C)$ methods (see e.g., [22], and [23]). In this work, we do not need a stochastic quadrature rule because the uncertainty information is already presented in discrete scenarios (that we weigh equally) from EPS forecasts; however, integrating other sources of uncertainty in future work may require the usage of a stochastic quadrature rule.

\section{B. The trajectory ensemble}

Given a quadrature rule and a given number of samples $N$, we define the trajectory ensemble associated to a control law $\mathbf{u}_{L}$ and a stopping criterion $s$ as the set of trajectories $\left\{\left(t_{f, k}, \mathbf{x}_{k}, \mathbf{u}_{k}\right)\right\}$ with $k \in\{1, \ldots, N\}$ such that the trajectory

\footnotetext{
${ }^{4}$ despite similarity in notation, this is not a stochastic differential equation because the random parameters are not random processes, i.e., are constant.
} 
$k$ is generated by the control and stopping rules with $\xi=\xi_{k}$ and the stopping criterion is met at $t=t_{f, k}$, i.e.

$$
\begin{gathered}
\frac{d}{d t} \mathbf{x}_{k}(t)=f\left(\mathbf{x}_{k}(t), \mathbf{u}_{L}\left(t, \mathbf{x}_{k}(t)\right), \xi_{k}(\omega), t\right) \\
s\left(t, \mathbf{x}_{k}(t)\right)<0, \forall t<t_{f} \\
s\left(t_{f, k}, \mathbf{x}_{k}\left(t_{f, k}\right)\right)=0
\end{gathered}
$$

We consider a virtual dynamical system whose state vector contains the state vectors of all the trajectories in the trajectory ensemble, which evolve each according to the dynamics in each scenario (i.e. for each value $\xi_{k}$ of $\xi$ ). Using this trajectory ensemble, the robust optimal control problem can be reformulated as a large deterministic OCP, where the $N$ trajectories are considered simultaneously.

\section{The "state-tracking" ROCP}

In previous literature employing this approach (see [17],[24], [25] or [26]), the control law is considered as only depen-dant on time $\mathbf{u}(\omega, t)=\mathbf{u}_{L}(t)$, thus leading to an "open-loop" control scheme. This "open-loop" formulation is, however, not a practical scheme for general optimal control problems. In some problems, the dynamic system could be unstable and the trajectories would diverge towards undesirable regions of the state space; in other (as the one we face in commercial aircraft trajectory optimization), we need to apply final conditions and/or have a unique path for some of the states.

Instead of looking for an optimal control, then, we will look for an optimal guidance; we designate some of the states as "tracked" states and we replace the unique controls $\mathbf{u}_{L}(t)$ that are applied identically in all scenarios by scenario-specific controls $\mathbf{u}_{k}(t)$ that ensure that the tracked states follow a unique trajectory for all likely values of the random variables (as long as it is feasible within the dynamics and constraints of the problem). In a real-world implementation, where the realized uncertainty would generally be a mix of the discrete scenarios that we are considering, we assume that the controls can be computed by existing controllers in order to track the calculated trajectory. In our context, the controls can be computed by the autopilot in order for the aircraft to follow a route at the calculated airspeeds and altitudes.

Let $\left\{i_{1}, \ldots, i_{q}\right\}$ be the indexes of the states we are interested in tracking (e.g. if we are tracking $x_{2}$ and $x_{5}, i_{1}=2$ and $i_{2}=5$ ). Let $e_{i}$ be the column vector that has a 1 at the position $i$; we define the matrix $E \in \mathbb{R}^{q \times n}$ as

$$
E=\left[\begin{array}{c}
e_{i_{1}}^{T} \\
\vdots \\
e_{i_{q}}^{T}
\end{array}\right]
$$

We define the problem as:

$$
\min J=\mathbb{E}\left[\phi\left(\mathbf{x}_{\mathbf{f}}\right)+\int_{t_{0}}^{t_{f}} \mathcal{L}(\mathbf{x}(\omega, t), u(\omega, t), t) d t\right]
$$

subject to the differential equations (III-B), the statetracking condition:

$$
E\left(\mathbf{x}\left(\omega_{1}, t\right)-\mathbf{x}\left(\omega_{2}, t\right)\right)=0, \forall t, \forall \omega_{1}, \omega_{2} \in \Omega
$$

the stopping rule $s(t, \mathbf{x}(t))=t-t_{f}$ and the boundary conditions:

$$
\begin{gathered}
\mathbf{x}\left(\omega, t_{0}\right)=\mathbf{x}_{0} \\
\mathbb{E}\left[\psi\left(\mathbf{x}\left(\omega, t_{f}\right)\right)\right]=0
\end{gathered}
$$

where $\psi$ is the function that represents the final conditions. As emphasized earlier, the controls are no longer unique as in the open-loop problem; they depend on the realization of $\xi(\omega)$. Here, the final conditions that depend only on the tracked states and the final time can be imposed exactly and not only in average. The corresponding discretization is

$$
\min J=\sum_{k=1}^{N} w_{k}\left[\phi\left(\mathbf{x}_{k}\left(t_{f}\right)\right)+\int_{t_{0}}^{t_{f}} \mathcal{L}\left(\mathbf{x}_{k}(t), \mathbf{u}_{k}(t), t\right) d t\right]
$$

subject to:

$$
\begin{gathered}
\dot{\mathbf{x}}_{k}=f\left(\mathbf{x}_{k}(t), \mathbf{u}_{k}(t), \xi_{k}, t\right), k \in\{1, \ldots, N\} \\
\mathbf{x}_{k}\left(t_{0}\right)=\mathbf{x}_{0}, k \in\{1, \ldots, N\} \\
E\left(\mathbf{x}_{k}(t)-\mathbf{x}_{1}(t)\right)=0, \forall k \in\{2, \ldots, N\} \\
\sum_{k=1}^{N} w_{k} \psi\left(\mathbf{x}_{k}\left(t_{f}\right)\right)=0
\end{gathered}
$$

\section{Application to aircraft robust trajectory optimization}

We look to find routes that minimize a weighted sum of average fuel consumption, flight time dispersion (weighted with parameter $d p$ as in dispersion penalty) and convection risk (weighted with parameter $c p$ as convection penalty). By changing the relative weight of $d p$ (assuming $c p=0$ ), we can obtain routes that are more efficient on average or routes that are more predictable. $d p=0$ means that we look for maximum average efficiency and higher values of $d p$ put more weight on dispersion, which we will define as the difference between the earliest and the latest arrival time. By changing the relative weight of $c p$ (assuming now $d p=0$ ), we can obtain routes that are more efficient on average or routes that are less risky in terms of convection (less probable to run into storms). Higher values of $c p$ put more weight on convective risk, and thus solution would try to avoid them.

The readers are referred to [5] for additional information and mathematical details of this methodology.

\section{Case Study}

\section{A. Description and Statement}

We consider an BADA3 A330 Aircraft model flying from the vertical of New York (-73.7789 deg, $40.6397 \mathrm{deg})$ to the vertical of Argel (3.2169 deg, $36.694 \mathrm{deg}$ ) at constant barometric altitude $200 \mathrm{hPa}$ and constant Mach number 0.82 (temperature is assumed to follow ISA and thus True Airspeed can be also considered constant). Initial mass has been set to 


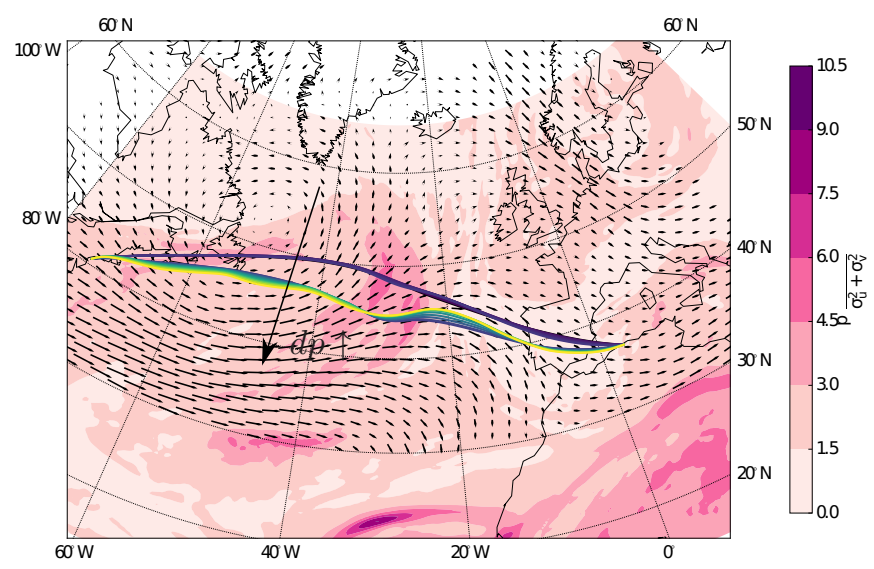

(a) Sweep dp $(\mathrm{cp}=0)$ on wind uncertainty map

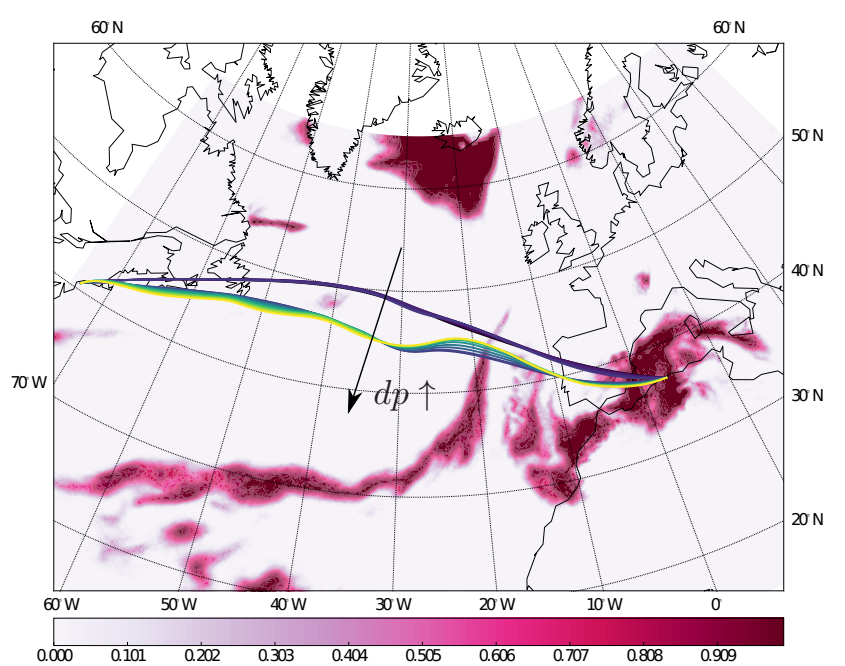

(c) Sweep dp $(\mathrm{cp}=0)$ on convective risk map

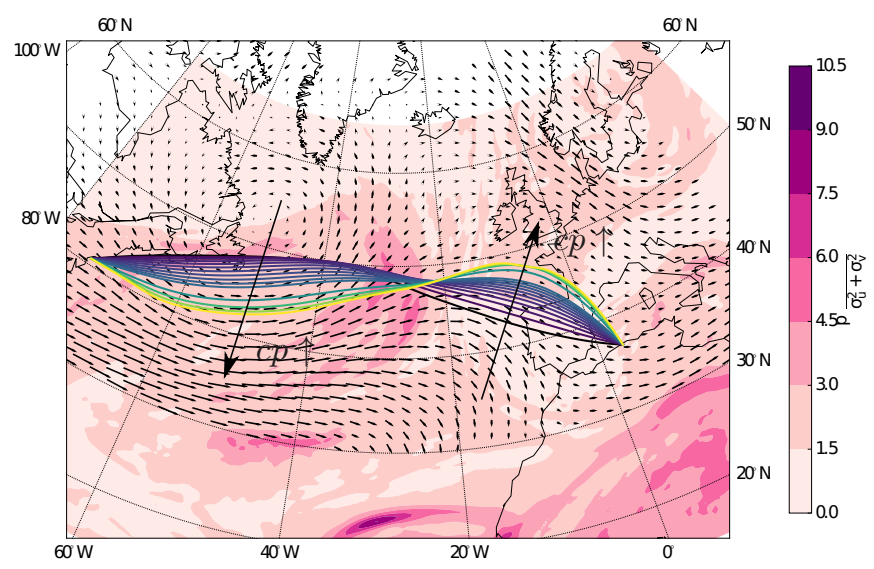

(b) Sweep cp $(\mathrm{dp}=0)$ on wind uncertainty risk map

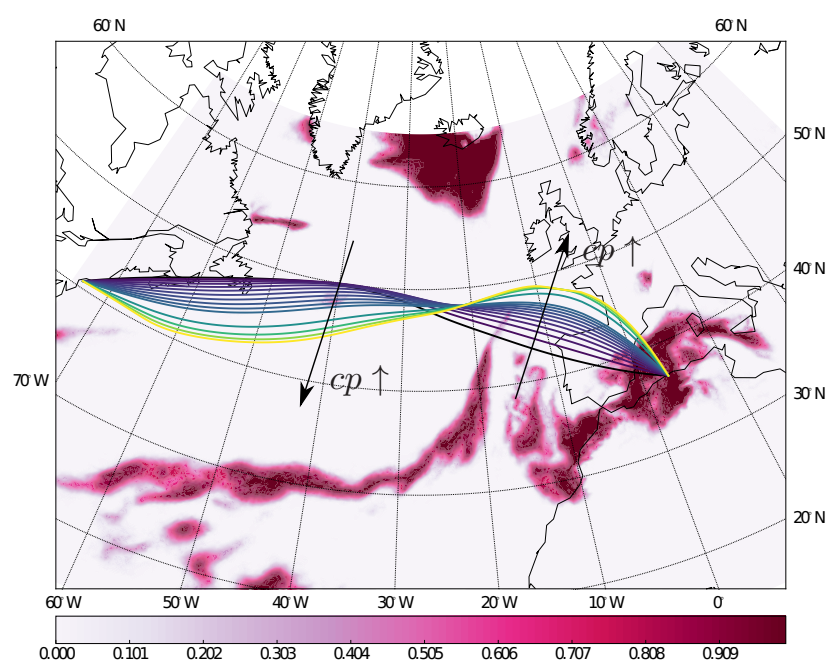

(d) Sweep cp $(\mathrm{dp}=0)$ on convective risk map

Figure 3. Optimal trajectories for $d p$ and $c p$ values. Higher brightness in the trajectory color indicates higher values of the penalty. Top figures depict the set of trajectories over a map with color regions of higher uncertainty, defined as $\sqrt{\sigma_{u}^{2}+\sigma_{v}^{2}}$, with $\sigma_{u}$ being the standard deviation of the $u$ component of wind across different members and $\sigma_{v}$ analogous for the v-component. Down figures depict the set of trajectories over a map with color regions of higher convective risk. Left trajectories are for $d p$ values ( 0 to 50) with $c p=0$. Right trajectories are for $c p$ values $(0$ to 0.03$)$ with $d p=0$.

200 tons. A free routing airspace is assumed for the sake of illustration. We use a forecast for a pressure of $200 \mathrm{hPa} 9$ hours in advance for the 19th of December, 2016 from the ECMWF ensemble, elaborated by the European Center for MediumRange Weather Forecasts (ECMWF) ${ }^{5}$ with 51 members. We rely on the Pyomo library as NLP interface [27] and IPOPT [28] as NLP solver.

\section{B. Results and discussion}

Figure 3 displays the geographical routes for different values of $d p$ and $c p$. It can be seen that routes computed with higher $d p$ (setting $c p=0$ ) tend to avoid the high uncertainty zone in the Atlantic in order to increase predictability, at the cost of taking a more indirect route that is longer on average. It can be also observed that routes computed with higher $c p$ (setting

${ }^{5}$ http://apps.ecmwf.int/datasets/ $d p=0)$ tend to reduce the exposure to convective risk zones, again at the cost of taking a more indirect route. The problem could have been solved for additional $c p$ - $d p$ pairs (including those with both values different than zero). It is important to remark that the exposure to convective risk areas could somehow turned into additional expected delay due to, for instance, (tactical) ATFM regulations or ATC advisories to avoid developed storms. This (relate $c p$ with $d p$ ) is however an open problem that we expect to face in the short term.

Figure 4.a shows the evolution of state and control variables along the average-min-fuel-optimal trajectory (corresponding to $d p=0$ and $c p=0$, the black line in 3). It can be seen that the spread in the ensemble times, headings, and ground speeds increases slightly when the aircraft crosses the area of high uncertainty in the middle of the North Atlantic (that can be seen in the left hand side Figures 3). Figure 4.b shows the evolution of state and control variables along the average-most predictable trajectory (corresponding to $d p=50$ and $c p=0$, 

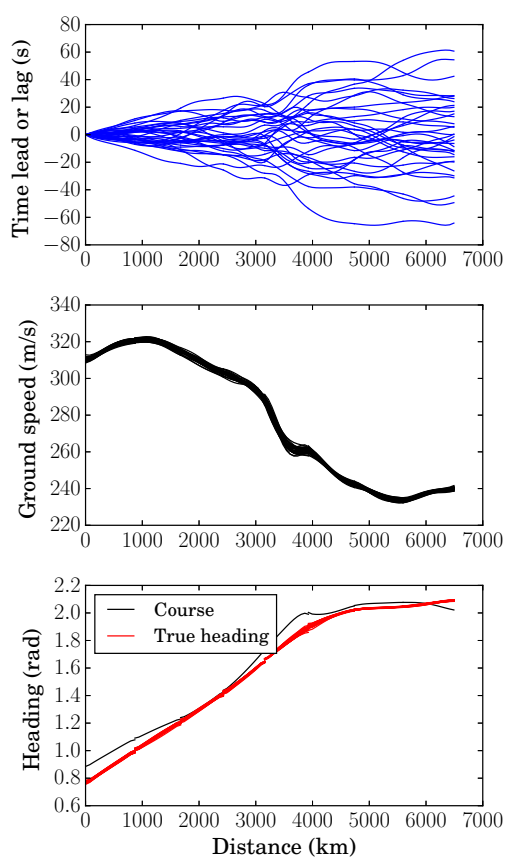

(a) min. fuel $(\mathrm{dp}=0 ; \mathrm{cp}=0)$
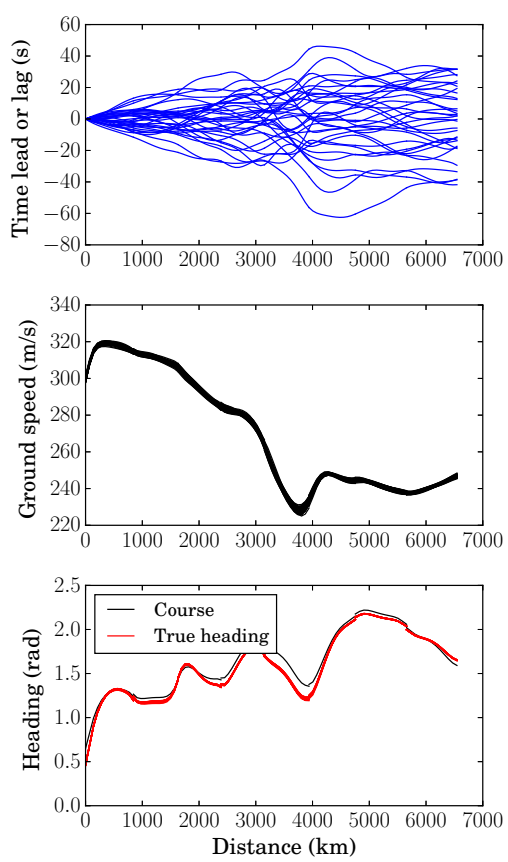

(b) max. predictability $(\mathrm{dp}=50 ; \mathrm{cp}=0)$
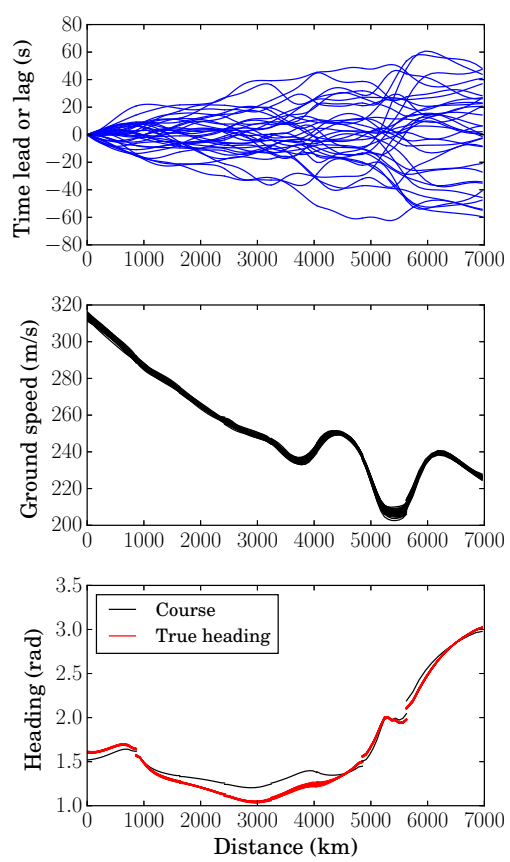

(c) min. convection risk $(\mathrm{dp}=0 ; \mathrm{cp}=0.03)$

Figure 4. State-space evolution of the variables. Time leads and lags are defined with respect to the average trajectory.

the yellow line in the left hand side trajectories of Fig.3). The spread in times and ground speeds are comparatively lower than in the previous case. Figure 4.c shows the evolution of state and control variables along the average-less convection exposure trajectory (corresponding to $d p=0$ and $c p=0.03$, the yellow line in the right hand side trajectories of Fig.3). In this case, minimizing time spread is not part of the objective and thus trajectories do not seek to avoid regions of wind uncertainty (this is why the time spread is rather high), but regions of convective risk (as it can be observed in Fig. 3).

Figure 5.c and 5.d present the different solutions for problems with different $d p-c p$ pair values.

As for quantitative indicators: For the minimum average fuel case $(d p=0)$, the time dispersion at the final fix is above 2 minutes, whereas for the maximum predictability case $(d p=50)$, the time dispersion at the final fix is slightly below 1.25 minutes. In other words, around one minutes reduction in time uncertainty could be achieved by flying the most predictable trajectory $(d p=50)$. This would be however at roughly $1500 \mathrm{~kg}$ of extra fuel burnt. Taking another point of the Pareto frontier (again of the subproblem with $c p=0$ ), the increase in predictability of about 0.75 minutes would result in $750 \mathrm{~kg}$ of fuel consumption. In any case, the Pareto frontier shows different possible solutions with trade-offs dispersionconsumption. Notice however that these numbers correspond to this particular case (route, day, and weather forecasts). ${ }^{6}$

${ }^{6}$ For instance in the case study solved in [4]) reductions in time dispersion due to wind phenomena of around 3 minutes were achieved.
Further studies should asses quantitative values in a more systematic fashion.

\section{CONCLUSiOnS AND FUTURE WORK}

A robust optimal control methodology has been used for computing robust optimal routes based on Ensemble Prediction Systems. Also, an approach to calculate risk of convection using Ensemble Prediction Systems has been presented. This risk, a necessary though not sufficient condition for the formation of storms, has been included in the objective functional of the robust optimal control problem. We have demonstrated its utility in studying trade-offs between efficiency, predictability (measured in terms of dispersion in the final time) and exposure to convention. We can conclude that by using this method, uncertainty (in this case due to wind) can not only be quantified, but also reduced by proposing alternative trajectories. Also, convective areas can be avoided at the cost of efficiency and predictability. This algorithms could be of course interesting for both flight dispatchers (demand side of the problema) and networks managers (capacity side of the problem) for the design of a more robust ATM system and, in turn, improve the ATM performance in terms of safety, capacity (delays), and environment.

\section{ACKNOWLEDGMENTS}

This work has been partially supported by project TBO-MET project (https://tbomet$\mathrm{h} 2020 . \mathrm{com} /$ ), which has received funding from the SESAR JU under grant agreement No 699294 under European Union's Horizon 2020 research and innovation programme.

\section{REFERENCES}

[1] E. Casado, C. Goodchild, and M. Vilaplana, "Sensitivity of trajectory prediction accuracy to aircraft performance uncertainty," in Proc. of the AIAAINFOTECH@AEROSPACE (I@A) CONFERENCE. 


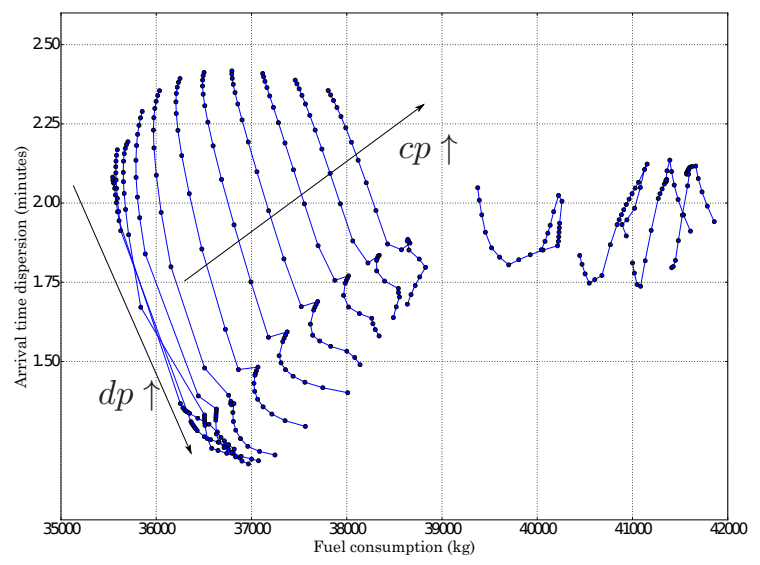

(a) Dispersion vs. fuel $\mathrm{dp} \& \mathrm{cp}$

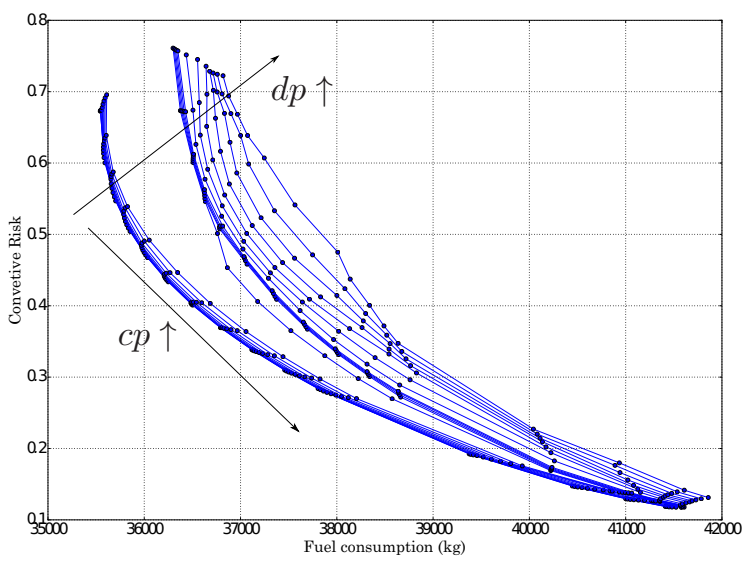

(b) Risk vs. fuel cp \& dp

Figure 5. Variable relations in the problem. Upper-Left Pareto frontier of the subproblem with $c p=0$.

[2] R. Vazquez and D. Rivas, "Propagation of initial mass uncertainty in aircraft cruise flight," Journal of Guidance, Control, and Dynamics, vol. 36, no. 2, pp. 415-429, 2013.

[3] E. Casado, M. Vilaplana, and C. Goodchild, "Sensitivity of continuous climb departure predictions to aircraft intent uncertainties," in 3th International Conference on Application and Theory of Automation in Command and Control Systems, 2013.

[4] D. Gonzalez-Arribas, M. Soler, and M. Sanjurjo, "Wind-based robust trajectory optimization using meteorological ensemble probabilistic forecasts," in SESAR Innovation Days 2016 (SIDs'16)., 2016.

[5] D. Gonzalez-Arribas, M. Soler, and M. Sanjurjo-Rivo, "Robust aircraft trajectory planning under wind uncertainty using optimal control," Journal of Guidance, Control, and Dynamics, Accepted for publication (2017).

[6] J. P. Hacker, E. S. Krayenhoff, and R. B. Stull, "Ensemble experiments on numerical weather prediction error and uncertainty for a north pacific forecast failure," Weather and forecasting, vol. 18, no. 1, pp. 12-31, 2003.

[7] P. Bauer, A. Thorpe, and G. Brunet, "The quiet revolution of numerical weather prediction," Nature, vol. 525, no. 7567, pp. 47-55, 2015.

[8] J. Cheung, J.-L. Brenguier, J. Heijstek, A. Marsman, and H. Wells, "Sensitivity of flight durations to uncertainties in numerical weather prediction," SESAR Innovation Days, 2014.

[9] J. Cheung, A. Hally, J. Heijstek, A. Marsman, and J.-L. Brenguier, "Recommendations on trajectory selection in flight planning based on weather uncertainty."

[10] R. Vazquez, D. Rivas, and A. Franco, "Stochastic analysis of fuel consumption in aircraft cruise subject to along-track wind uncertainty," Aerospace Science and Technology, vol. 66, pp. 304-314, 2017.

[11] K. Legrand, S. Puechmorel, D. Delahaye, and Y. Zhu, "Aircraft trajectory planning under wind uncertainties," in Digital Avionics Systems Conference (DASC), 2016 IEEE/AIAA 35th. IEEE, 2016, pp. 1-9.

[12] R. Carbone, J. Tuttle, D. Ahijevych, and S. Trier, "Inferences of predictability associated with warm season precipitation episodes," Journal of the Atmospheric Sciences, vol. 59, no. 13, pp. 2033-2056, 2002.

[13] G. Ellrod and G. Field, "The characteristics and prediction of gulf stream thunderstorms," in Conference on Weather Forecasting and Analysis, 10 th, Clearwater Beach, FL, 1984, pp. 15-21.

[14] M. Tiedtke, "A comprehensive mass flux scheme for cumulus parameterization in large-scale models," Monthly Weather Review, vol. 117, no. 8, pp. 1779-1800, 1989.

[15] P. Bechtold, M. Köhler, T. Jung, F. Doblas-Reyes, M. Leutbecher, M. J. Rodwell, F. Vitart, and G. Balsamo, "Advances in simulating atmospheric variability with the ecmwf model: From synoptic to decadal time-scales," Quarterly Journal of the Royal Meteorological Society, vol. 134, no. 634, pp. 1337-1351, 2008.

[16] P. Bechtold, N. Semane, P. Lopez, J.-P. Chaboureau, A. Beljaars, and N. Bormann, "Representing equilibrium and nonequilibrium convection in large-scale models," Journal of the Atmospheric Sciences, vol. 71, no. 2, pp. 734-753, 2014.
[17] I. M. Ross, R. J. Proulx, M. Karpenko, and Q. Gong, "Riemann-stieltjes optimal control problems for uncertain dynamic systems," Journal of Guidance, Control, and Dynamics, pp. 1-13, 2015.

[18] I. H. Sloan and H. Woźniakowski, "When are quasi-monte carlo algorithms efficient for high dimensional integrals?" Journal of Complexity, vol. 14, no. 1, pp. 1-33, 1998.

[19] S. Tezuka, "On the necessity of low-effective dimension," Journal of Complexity, vol. 21, no. 5, pp. 710-721, 2005.

[20] R. Cools and P. Rabinowitz, "Monomial cubature rules since "stroud": a compilation," Journal of Computational and Applied Mathematics, vol. 48, no. 3, pp. 309-326, 1993.

[21] R. Cools, "Monomial cubature rules since "stroud": a compilation—part 2," Journal of Computational and Applied Mathematics, vol. 112, no. 1, pp. 21-27, 1999.

[22] D. Xiu, Numerical methods for stochastic computations: a spectral method approach. Princeton University Press, 2010.

[23] A. O'Hagan, "Polynomial chaos: A tutorial and critique from a statistician's perspective," 2013.

[24] X. Li, P. B. Nair, Z. Zhang, L. Gao, and C. Gao, "Aircraft robust trajectory optimization using nonintrusive polynomial chaos," Journal of Aircraft, vol. 51, no. 5, pp. 1592-1603, 2014.

[25] J. Fisher and R. Bhattacharya, "Optimal trajectory generation with probabilistic system uncertainty using polynomial chaos," Journal of dynamic systems, measurement, and control, vol. 133, no. 1, p. 014501, 2011.

[26] T. C. Flanzer, G. C. Bower, and I. M. Kroo, "Robust trajectory optimization for dynamic soaring," in AIAA Guidance, Navigation, and Control Conference, 2012, p. 4603.

[27] W. E. Hart, J.-P. Watson, and D. L. Woodruff, "Pyomo: modeling and solving mathematical programs in python," Mathematical Programming Computation, vol. 3, no. 3, pp. 219-260, 2011.

[28] A. Wächter and L. T. Biegler, "On the implementation of an interiorpoint filter line-search algorithm for large-scale nonlinear programming," Mathematical programming, vol. 106, no. 1, pp. 25-57, 2006.

\section{COPYRight StATEMENT}

The authors confirm that they, and/or their company or institution, hold copyright of all original material included in their paper. They also confirm they have obtained permission, from the copyright holder of any third party material included in their paper, to publish it as part of their paper. The authors grant full permission for the publication and distribution of their paper as part of the EIWAC2017 proceedings or as individual off-prints from the proceedings. 